\title{
РАЗРАБОТКА ПОДХОДА К ПРОЕКТИРОВАНИЮ И РАСЧЕТУ БЕЗВОЗДУШНОГО КОЛЕСА ЭЛЕКТРИЧЕСКОГО САМОКАТА
}

\author{
А. Т. Волочко ${ }^{1}$, С. Н. Янкевич² \\ ${ }^{1}$ Д. т. н., профрессор, начальник отдела, заведующий лабораторией микрокристаллических и аморфнных материалов \\ физико-технического института Национальной академии наук Беларуси, Минск, Беларусь, e-mail: phti@belhost.by \\ ${ }_{2}^{2}$ Аспирант, нач. научно-исследовательского отдела, \\ ОАО «Приборостроительный завод Оптрон», Минск, Беларусь, e-mail: com@optron.by
}

\begin{abstract}
Реферат
Предложена методика расчета безвоздушного колеса электрического самоката, основанная на применении системного подхода. Выполнен анализ безвоздушного колеса как механической системы с учетом внешнего нагружения, представлен расчет внешних нагрузок и жесткости безвоздушного колеса и обоснование его конструктивного исполнения, предложена методика выбора конструктивных параметров спиц безвоздушного колеса электрического самоката и выполнен синтез конструкции безвоздушного колеса с помощью метода конечных элементов. Приведенные зависимости учитывают как конструктивные параметры самоката и применяемого колеса, так и массу водителя. Они могут быть использованы при анализе нагруженности безвоздушного колеса, объединяющего функции колеса и амортизатора при разработке методики сравнительных экспериментальных исследований безвоздушных колес для самокатов.
\end{abstract}

Ключевые слова: конечно-элементное моделирование, электротранспорт, безвоздушное колесо, математическая модель, иерархическая модель, электросамокат.

\section{DEVELOPMENT OF AN APPROACH TO THE DESIGN AND CALCULATION OF AN AIRLESS WHEEL FOR AN ELECTRIC SCOOTER}

\section{A. T. Volochko, S. N. Yankevich}

\section{Abstract}

A method of calculating the airless wheel of an electric scooter, based on the application of a systematic approach, is proposed. The analysis of the airless wheel as a mechanical system taking into account external loading is performed, the calculation of external loads and stiffness of the airless wheel and the justification of its design are presented, the method of selecting the design parameters of the spokes of the airless wheel of an electric scooter is proposed, and the synthesis of the design of the airless wheel is performed using the finite element method. These dependencies take into account both the design parameters of the scooter and the wheel used, and the weight of the driver. They can be used in the analysis of the load of an airless wheel that combines the functions of a wheel and a shock absorber in the development of a methodology for comparative experimental studies of airless wheels for scooters.

Key words: finite element modeling, electric transport, airless wheel, mathematical model, hierarchical model, electric scooter.

\section{Введение}

В настоящее время наибольшей популярностью у покупателей индивидуального электротранспорта пользуются электрические самокаты. Так, в июне 2020 года в торговых сетях Российской Федерации рост продаж электросамокатов составил $25 \%$ в штуках и $70 \%$ в деньгах по сравнению с июнем 2019 года. На интернет-площадках КНР скачок роста пришелся на май - в первые три недели месяца продажи выросли на $350 \%$ по сравнению с апрелем 2020 года [1]. Это обусловлено рядом факторов: портативность, невысокая цена, запас хода, расход электроэнергии и т. д. Кроме того, электросамокаты могут разгрузить дороги и решить так называемую проблему последней мили - помочь добраться от дома до метро не на машине, а на транспорте, не создающем пробок и не требующем парковочного места [2].

В 2017 году мировой рынок электросамокатов был оценен в $\$ 630$ млн (он составил $8 \%$ от всего рынка двухколёсного электротранспорта без учета электробайков), однако согласно прогнозам, доля электросамокатов к 2024 году вырастет до 14,5\%.

Конструкция электрического самоката представлена на рисунке 1.

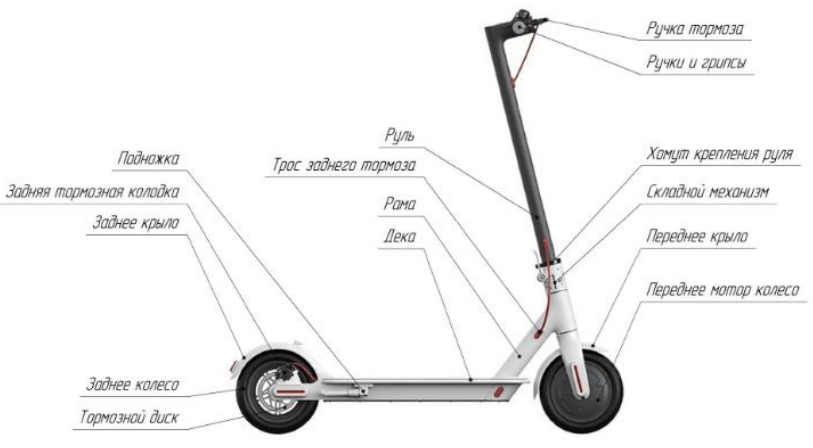

Рисунок 1 - Конструкция электрического самоката
Конструкция электросамокатов, будучи принципиально сравнительно простой, вместе с тем оставляет немало направлений для современного научного поиска. Являясь одним из популярных транспортных средств, применение в их конструкции новейших достижений науки и технологий очень актуально. Это невозможно без выполнения анализа нагруженности как самого самоката, так и его компонентов. В частности особый интерес, в виду большого количества нареканий пользователей, вызывают пневматические шины, применяемые в их конструкции, а именно их малый ресурс, подверженность повреждениям и т. д. Основной массы этих недостатков лишены безвоздушные шины, в которых гибкие упругие элементы выполняют функции воздуха в пневматических шинах. Однако их применение в конструкции индивидуального электротранспорта сопряжено с необходимостью решения ряда физических и математических задач.

Цель работы заключается в разработке методики расчета и проектирования безвоздушного колеса электрического самоката, основанной на применении системного подхода.

Задачами данной работы являются:

- разработать иерархическую модель системы безвоздушного колеса с применением системного подхода;

- выполнить анализ безвоздушного колеса как механической системы с учетом внешнего нагружения и жесткости колеса;

- создать модель конструкции безвоздушного колеса электрического самоката с использованием метода конечных элементов.

\section{Системный подход к разработке модели безвоздушного} колеса электрического самоката

На рисунке 2 показана иерархическая модель системы «безвоздушное колесо». Представление безвоздушного колеса как сложной системы посредством применения системного подхода позволяет, с одной стороны, разделить его проектирование на этапы, с другой учесть существующие связи между подсистемами, определяющие его работоспособность. Вместе с тем, излишняя детализация и конкретизация может не только значительно затруднить, но и сделать подобный 
анализ практически невозможным. Поэтому можно констатировать, что при моделировании такой системы нецелесообразно создавать одну универсальную модель, которая могла бы воспроизводить как действие системы в целом, так и отдельных ее подсистем.

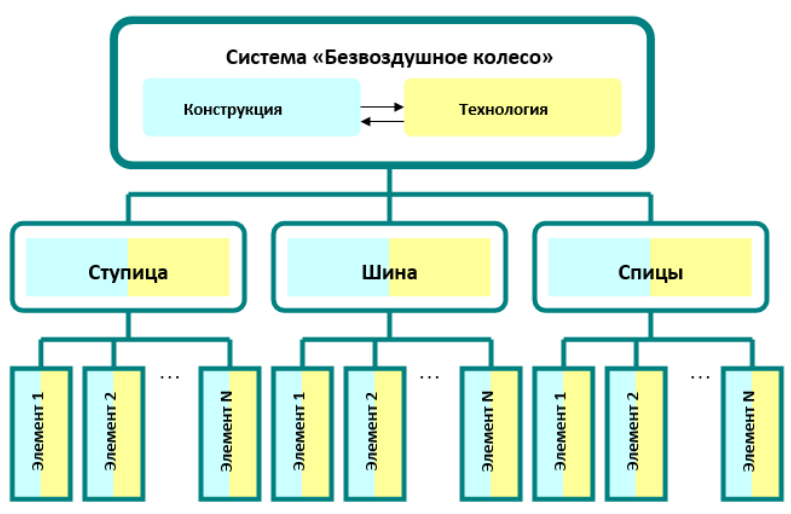

Рисунок 2 - Иерархическая модель системы «Безвоздушное колесо»

Несомненно, одной из важнейших предпосылок является учет влияния при рядовой эксплуатации как внешнего воздействия, так и конструктивно-технологических особенностей объекта. Именно этот аспект во многом определяет неоднозначность и неопределенность анализа и исходов. Вместе с тем, отказ от учета этого фактора, очевидно, может внести существенную погрешность в предполагаемые выводы исследования.

\section{Расчет внешних нагрузок}

Несущая способность безвоздушных шин в значительной мере определяется механическими характеристиками упругих элементов и, прежде всего, радиальной жесткостью.

Расчетная схема нагрузок представлена на рисунке 3.

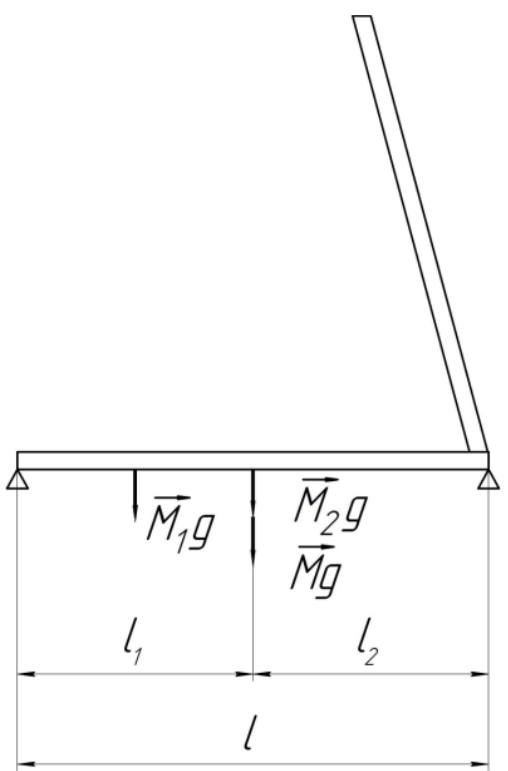

Рисунок 3 - Расчетная схема нагруженности электрического самоката

Пусть масса водителя равна $M_{1}$, а масса электрического самоката равна $M 2$. Тогда, согласно закону Гука, путем математических преобразований получим, что величина х (мм), показывающая, насколько сожмется подвеска под действием массы водителя и самоката, составит

- при наличии сиденья $\quad x=\frac{H \cdot g}{h \cdot k}\left(M_{1}+\frac{l_{1}}{l} \cdot M_{2}\right)$,

- при отсутствии сиденья $\quad x=\frac{H \cdot g}{h \cdot k}\left(\frac{1}{2} M_{1}+\frac{l_{1}}{l} \cdot M_{2}\right)$. где $k$ - коэффициент упругости (Н/мм);

I - длина деки самоката,

$I=l_{1}+l_{2}, I_{1}$ и $I_{2}-$ расстояния от краев деки до центра тяжести;

H-ход (мм) десормации колеса,

$h$ - сжатие колеса (мм),

$M_{1}$ - масса водителя;

$M_{2}$ - масса электрического самоката;

$M$ - общая масса.

Данная зависимость учитывает как конструктивные параметры самоката и применяемого колеса, так и массу водителя. Она может быть использована как при анализе нагруженности безвоздушного колеса, объединяющего функции колеса и амортизатора, так и при разработке методики сравнительных экспериментальных исследований безвоздушных колес для самокатов.

Анализ жесткости безвоздушного колеса электрического самоката

Очевидно, что реализация внутреннего демпфирования неразрывно связана, прежде всего, с оптимизацией формы спиц безвоздушного колеса. Применение спицы дугообразной формы или спицы, представляющей собой аналог сотовой структуры, могло бы решить проблему, но это не позволяет полностью реализовать существующие возможности для решения задачи, о чем свидетельствует отсутствие на массовом рынке подобных решений.

В качестве приемлемого решения может быть принято конструктивное исполнение спиц в виде витых пружин S-образной фрормы (рисунок 4). Такая конструкция обусловлена необходимостью обеспечения боковой жесткости колеса при минимальном количестве деталей и узлов изделия. Применение витых пружин в подобных конструкциях ведет к необходимости наличия направляющих в конечном изделии. Однако, благодаря своей форме, плоские витые пружины выполняют функцию бокового ребра жесткости.

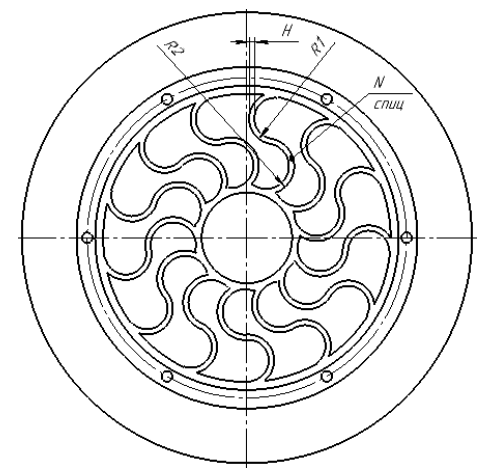

Рисунок 4 - Предлагаемая конструкция безвоздушного колеса

Схема нагружения $i$-того радиального элемента безвоздушной шины радиальной нагрузкой Р представлена на рисунке 5 .

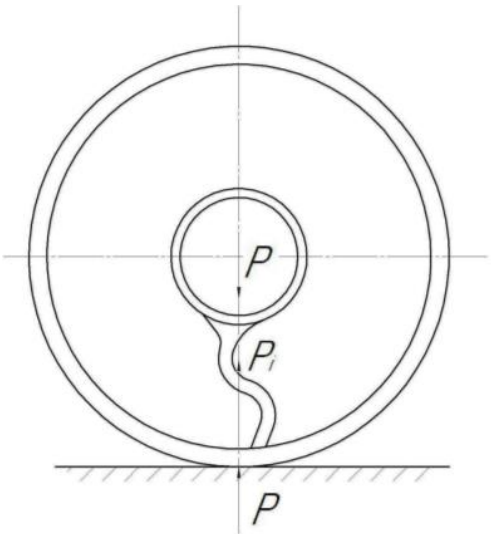

Рисунок 5 - Схема нагружения безвоздушной шины c $N$ упругими элементами радиальной нагрузкой $\mathrm{P}$ 
Предположим, что упругие элементы рассматриваются как непрерывная упругая среда. В этом случае для любой точки обода сила, действующая со стороны упругого элемента, будет пропорциональна радиальному смещению соответствующей точки обода $\omega$. Так, для $i$-го упругого элемента (рисунок 5) сила $P_{i}$ будет связана с радиальным перемещением точки обода $\omega_{i}$ соотношениями

$$
P_{i}=C \cdot \omega_{i}, i=\overline{1, N},
$$

где $\boldsymbol{C}$ - радиальная жесткость упругого элемента, $\mathrm{H} / \mathrm{M}$.

Схема нагружения упругого элемента показана на рисунке 6.

Для определения радиальной жесткости упругого элемента необходимо рассчитать перемещение сечения $A$ под действием силы $P_{i}$ в направлении этой силы. Это перемещение можно определить с помощью теоремы Кастелиано [3].

$$
\omega_{i}=\frac{\partial U}{\partial P_{i}}
$$

где $U$ - потенциальная энергия упругого элемента.

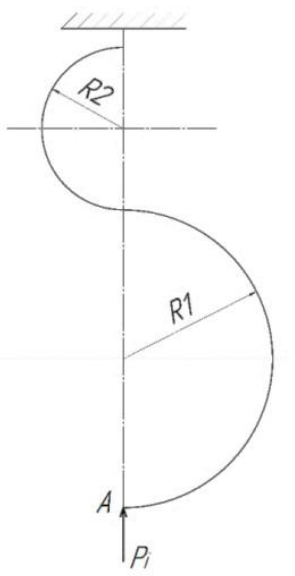

Рисунок 6 - Схема нагружения упругого элемента безвоздушной шины

Для упругого элемента, у которого основную роль играют изгибные напряжения, перемещения вследствие растяжения и сдвига так же малы по сравнению с перемещениями изгиба, как и энергия растяжения и сдвига по сравнению с энергией изгиба.

Поэтому выражение для потенциальной энергии изгиба элемента (с учетом постоянного поперечного сечения элемента по длине) будет иметь вид

$$
U=\frac{\int_{i} M_{P_{i}}^{2} d z}{2 E J},
$$

где $d z$ - длина элементарного участка упругого элемента;

$M_{P i}-$ функция изгибающего момента от действия силы $P_{i}$;

$E$ - модуль упругости упругого элемента;

$J$ - момент инерции сечения;

I - интегрирование осуществляется по длине бруса.

Из выражений (2)-(4) определяется радиальная жесткость $C_{i}$ упругого элемента

$$
C_{i}=\frac{P_{i}}{\frac{\partial U}{\partial P_{i}}}=\frac{P_{i}}{\omega}
$$

где $P_{i}$ - действующая радиальная сила, $\omega$ - радиальное смещение соответствующей точки обода.
Исследование напряженного состояния упругого элемента и обода безвоздушной шины под действием радиальной силы (рисунок 6) можно осуществить с помощью метода, изложенного Феодосьевым В. И. [4].

Если спицы рассматривать как непрерывную упругую среду, то для любой точки обода сила, действующая со стороны спиц, будет пропорциональна радиальному смещению соответствующей точки обода $\omega$ (при этом, очевидно, форма спиц - прямолинейная или s-образная не имеет никакого значения). Таким образом, данная задача представляет собой аналог задачи о расчете кольца с упругим основанием. На единицу длины обода приходится $N / 2 \pi R$ спиц. Со стороны каждой спицы на обод действует сила $E F \omega / I$, где I - длина прямолинейного отрезка, связывающего крайние точки $S$-образной спицы $(I \sim R), F-$ площадь поперечного сечения спицы.

На единицу обода, таким образом, действует сила

$$
\frac{E \cdot F \cdot n}{2 \pi R^{2}} \omega=k \omega,
$$

откуда

$$
k=\frac{E \cdot F \cdot n}{2 \pi R^{2}} .
$$

Составим дифференциальное уравнение упругой линии кольца. За независимую переменную выберем угол $\varphi$, отсчитываемый от вершины кольца (рисунок 7).

Из кольца выделим элементарный участок длины $R d \varphi$ и в произведенных сечениях приложим внутренние силы $N, Q$ и $M$. Со стороны спиц на этот участок будет действовать сила $k \omega R d \varphi$. Составим уравнения равновесия для этого элементарного участка. Проектируем все силы на радиальную ось. Тогда получим

$$
\frac{\partial Q}{\partial \varphi}=N+k R \omega
$$
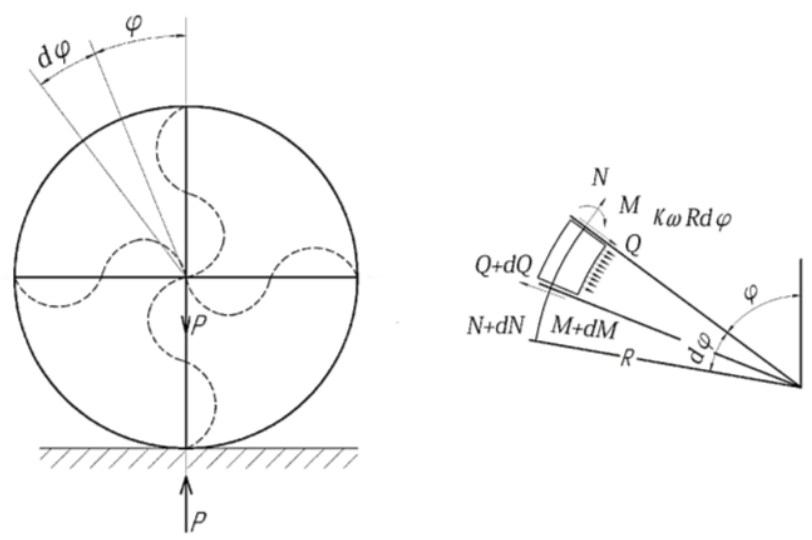

Рисунок 7 - Определение независимой переменной $\varphi$

Условие равенства нулю суммы проекций всех сил на ось, касательную к дуге круга, дает

$$
\frac{\partial N}{\partial \varphi}+Q=0 .
$$

Приравняем к нулю сумму моментов сил относительно точки 0

$$
R \frac{\partial N}{\partial \varphi}+\frac{\partial M_{x}}{\partial \phi}=0
$$

и исключаем из этих уравнений $Q$ и $N$. Тогда имеем

$$
k \cdot R^{2} \frac{\partial \omega}{\partial \varphi}=\frac{\partial M_{x}}{\partial \varphi}+\frac{\partial^{3} M_{x}}{\partial \varphi^{3}} .
$$


Изменение кривизны $\Delta(1 / \rho)$ связано с изгибающим моментом $M_{x}$ следующим соотношением

$$
M_{x}=E \cdot J \cdot \Delta\left(\frac{1}{\rho}\right),
$$

но, как известно,

$$
\Delta=\left(\frac{1}{\rho}\right)=-\left(\frac{\omega}{R^{2}}+\frac{1}{R^{2}} \cdot \frac{\partial^{2} \omega}{\partial \varphi^{2}}\right) .
$$

Так как при положительном перемещении $\omega$, направленном от центра круга, кривизна кольца уменьшается, в правой части этого выражения стоит знак минус. Изменение кривизны в этом выражении состоит из двух величин. Первое слагаемое $\omega / R^{2}$ соответствует изменению кривизны за счет простого расширения кольца. Второе слагаемое

$$
\frac{1}{R^{2}} \cdot \frac{\partial^{2} \omega}{\partial \varphi^{2}}=\frac{\partial^{2} \omega}{\partial s^{2}}
$$

представляет собой обычное изменение кривизны, которое наблюдается в прямом брусе.

После подстановки $M$ дифференциальное уравнение приобретает следующий окончательный вид:

$$
\frac{\partial^{5} \omega}{\partial \varphi^{5}}+2 \frac{\partial^{3} \omega}{\partial \varphi^{3}}+a^{2} \frac{\partial \omega}{\partial \varphi}=0
$$

где

$$
a^{2}=\frac{R^{4} k}{E J}+1
$$

Решением этого уравнения будет

$$
\begin{aligned}
& \omega=C_{0}+C_{1} \operatorname{ch} \alpha \varphi \cdot \cos \beta \varphi+C_{2} \operatorname{sh} \alpha \varphi \cdot \sin \beta \varphi+ \\
& +C_{3} \operatorname{ch} \alpha \varphi \cdot \sin \beta \varphi+C_{4} \operatorname{sh} \alpha \varphi \cdot \cos \beta \varphi
\end{aligned}
$$

где

$$
\begin{gathered}
\alpha=\sqrt{\frac{a-1}{2}}, \\
\beta=\sqrt{\frac{a+1}{2}} .
\end{gathered}
$$

Так как кольцо деформируется симметрично относительно вертикальной оси, функция $\omega$ должна быть четной, т.е. при перемене знака при $\varphi$ с плюса на минус должна остаться неизменной. Поэтому произвольные постоянные $C_{3}$ и $C_{4}$, стоящие при нечетных функциях, полагаем равными нулю.

Остальные постоянные определяются из следующих условий:

а) при $\varphi=\pi \frac{\partial \omega}{\partial \varphi}=0$;

б) при $\varphi=\pi Q=-\frac{P}{2}$;

в) $\int_{0}^{\pi} \omega \partial \varphi=0$.

Последнее условие означает, что при нагружении колеса верхняя и нижняя точки остаются на одной вертикали. Действительно, если рассмотреть элемент обода колеса до и после деформации (рисунок 8), то нетрудно установить, что условие его нерастяжимости можно представить в виде $\partial v+\omega \partial \varphi=0$

где $U$ - перемещение по касательной к дуге контура, или $v=-\int \omega \partial \varphi$

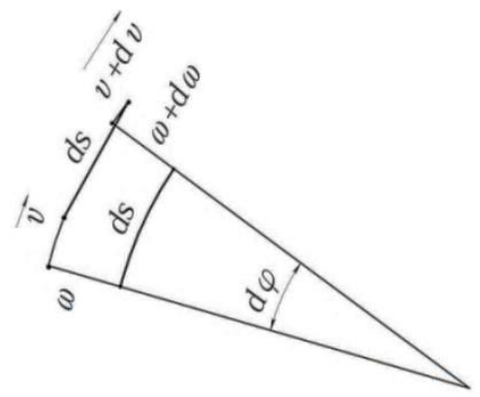

Рисунок 8 - Элемент обода до и после деформации

Так как смещение по касательной в точках $\varphi=0$ и $\varphi=\pi$ отсутствует, то отсюда и вытекает условие в).

При $C_{3}=C_{4}=0$ выражения для изгибающего момента

$$
M_{x}=-\frac{E J}{R^{2}}\left(\omega+\frac{\partial^{2} \omega}{\partial \varphi^{2}}\right)
$$

и поперечной силь

$$
Q=\frac{1}{R} \cdot \frac{\partial M_{x}}{\partial \varphi}
$$

принимают вид

$$
\begin{aligned}
& M_{x}=-\frac{E J}{R^{2}}\left(C_{0}-2 \cdot \alpha \cdot \beta \cdot C_{1} \operatorname{sh} \alpha \varphi \cdot \sin \beta \varphi+\right. \\
& \left.+2 \cdot \alpha \cdot \beta \cdot C_{2} \operatorname{ch} \alpha \varphi \cdot \cos \beta \varphi\right) ; \\
& Q=2 \cdot \alpha \cdot \beta \cdot \frac{E J}{R^{2}}\left[\begin{array}{l}
\left(\alpha \cdot C_{1}+\beta \cdot C_{2}\right) \operatorname{ch} \alpha \varphi \cdot \sin \beta \varphi+ \\
+\left(\beta \cdot C_{1}+\alpha \cdot C_{2}\right) \operatorname{sh} \alpha \varphi \cdot \cos \beta \varphi
\end{array}\right] .
\end{aligned}
$$

Раскрывая граничные условия а), б) и в), получаем

a) $C_{1}[\alpha \cdot \operatorname{sh} \alpha \pi \cdot \cos \beta \pi+\beta \cdot \operatorname{ch} \alpha \pi]+$ a) $+C_{2}[\beta \cdot \operatorname{sh} \alpha \pi \cdot \cos \beta \pi+\alpha \cdot \operatorname{ch} \alpha \pi \cdot \sin \beta \pi]=0$;

$$
C_{1}[\alpha \cdot \operatorname{sh} \alpha \pi \cdot \cos \beta \pi+\beta \cdot \operatorname{ch} \alpha \pi]+
$$

б) $+C_{2}[\beta \cdot \operatorname{sh} \alpha \pi \cdot \cos \beta \pi+\alpha \cdot \operatorname{ch} \alpha \pi \cdot \sin \beta \pi]=-\frac{P}{4 E J} \cdot \frac{R^{3}}{\alpha \beta}$; $C_{0} \pi+\frac{C_{1}}{\alpha^{2}+\beta^{2}}[\alpha \cdot \operatorname{sh} \alpha \pi \cdot \cos \beta \pi+\beta \cdot \operatorname{ch} \alpha \pi \cdot \sin \beta \pi]+$ B) $+\frac{C_{2}}{\alpha^{2}+\beta^{2}}[\alpha \cdot \operatorname{ch} \alpha \pi \cdot \sin \beta \pi-\beta \cdot \operatorname{sh} \alpha \pi \cdot \cos \beta \pi]=0$

Решив эти уравнения, получим

$$
\begin{aligned}
& C_{0}=\frac{P R^{3}}{2 \pi \cdot \alpha^{2} E J}, \\
& C_{1}=-\frac{P R^{3} \cdot(\alpha \cdot \operatorname{ch} \alpha \pi \cdot \sin \beta \pi+\beta \cdot \operatorname{sh} \alpha \pi \cdot \cos \beta \pi)}{4 E J \cdot \alpha \cdot \beta \cdot a\left(\operatorname{sh}^{2} \alpha \pi+\sin ^{2} \beta \pi\right)}, \\
& C_{2}=\frac{P R^{3} \cdot(\alpha \cdot \operatorname{sh} \alpha \pi \cdot \cos \beta \pi-\beta \cdot \operatorname{ch} \alpha \pi \cdot \sin \beta \pi)}{4 E J \cdot \alpha \cdot \beta \cdot a\left(\operatorname{sh}^{2} \alpha \pi+\sin ^{2} \beta \pi\right)} .
\end{aligned}
$$


Окончательно выражения для $\omega$ и $M_{x}$ принимают вид:

$$
\begin{gathered}
\omega=\frac{P \cdot R^{3}}{4 \alpha \cdot \beta \cdot E \cdot J}\left(\frac{2 \alpha \cdot \beta}{\pi \cdot \alpha^{2}}-A \cdot \operatorname{ch} \alpha \varphi \cdot \cos \beta \varphi+B \cdot \operatorname{sh} \alpha \varphi \cdot \sin \beta \varphi\right), \\
M_{x}=-\frac{P R}{2}\left(\frac{1}{\pi \cdot a^{2}}+A \cdot \operatorname{sh} \alpha \varphi \cdot \sin \beta \varphi+B \cdot \operatorname{ch} \alpha \varphi \cdot \cos \beta \varphi\right),
\end{gathered}
$$

где

$$
\begin{aligned}
& A=\frac{\alpha \cdot \operatorname{ch} \alpha \pi \cdot \sin \beta \pi+\beta \cdot \operatorname{sh} \alpha \pi \cdot \cos \beta \pi}{a\left(\operatorname{sh}^{2} \alpha \pi+\sin ^{2} \beta \pi\right)}, \\
& B=\frac{\alpha \cdot \operatorname{sh} \alpha \pi \cdot \cos \beta \pi+\beta \cdot \operatorname{ch} \alpha \pi \cdot \sin \beta \pi}{a\left(\operatorname{sh}^{2} \alpha \pi+\sin ^{2} \beta \pi\right)} .
\end{aligned}
$$

Усилие, приходящееся на одну спицу, будет, очевидно, равно

$$
P_{i}=\frac{E F}{R} \omega
$$

Выражение (5) при этом примет вид

$$
C=\frac{4 \alpha \cdot \beta \cdot E \cdot J}{R^{3}\left(\frac{2 \alpha \cdot \beta}{\pi \cdot a^{2}}-A \cdot \operatorname{ch} \alpha \varphi \cdot \cos \beta \varphi+B \cdot \operatorname{sh} \alpha \varphi \cdot \sin \beta \varphi\right)} .
$$

Из (11)-(12) следует, что

$$
R=2 \sqrt{\frac{\alpha \cdot \beta \cdot J}{F\left(\frac{2 \alpha \cdot \beta}{\pi \cdot \alpha^{2}}-A \cdot \operatorname{ch} \alpha \varphi \cdot \cos \beta \varphi+B \cdot \operatorname{sh} \alpha \varphi \cdot \sin \beta \varphi\right)}} .
$$

С учетом того, что момент инерции прямоугольного сечения, имеющего толщину $H$ и ширину $L$, определяется формулой $J=\frac{H \cdot L^{3}}{12}$, а $F=H \cdot L$, выражение (13) принимает вид

$$
R=L \cdot \sqrt{\frac{\alpha \cdot \beta}{3\left(\frac{2 \alpha \cdot \beta}{\pi \cdot \alpha^{2}}-A \cdot \operatorname{ch} \alpha \varphi \cdot \cos \beta \varphi+B \cdot \operatorname{sh} \alpha \varphi \cdot \sin \beta \varphi\right)}}
$$

С учетом (11) и (14) жесткость С можно представить в виде

$$
C=\frac{E \cdot H \cdot L}{2 \cdot R} \cdot \sqrt{\frac{3\left(\frac{2 \alpha \cdot \beta}{\pi \cdot \alpha^{2}}-A \cdot \operatorname{ch} \alpha \varphi \cdot \cos \beta \varphi+B \cdot \operatorname{sh} \alpha \varphi \cdot \sin \beta \varphi\right)}{\alpha \cdot \beta}},
$$

где $H$ - толщина спиц колеса, $L$ - ширина спиц колеса.

Таким образом, выбор конструктивных параметров спиц безвоздушного колеса электрического средства персональной мобильности, основанный на применении аналитических методов исследования, может быть использован в качестве исходных данных при последующем применении численных методов исследования (метода конечных элементов).

Разработка конструкции безвоздушного колеса электрического самоката с использованием метода конечных элементов.

Следует отметить, что обычно требования к сложной технической системе в целом, а также ее подсистем и деталей, основанные только на инженерной практике и опыте эксплуатации, являются не только самым простым, но и наиболее распространенным подходом [5]. Показатели сложной технической системы, основанные на анализе имеющейся статистической информации по уже существующим объектам, близким по показателям к существующему образцу, являются в значительной степени прогнозом для технического совершенствования исследуемой конструкции. Однако такой прогноз в своей основе опирается на данные, полученные при эксплуатации.
Аналогичные подходы могут быть применены при анализе подсистем и деталей безвоздушного колеса.

На практике при расчете современных узлов конструкций наиболее полная математическая модель обычно не применяется, вместо этого используется инженерный опыт для ответа на вопрос, является ли выбранная математическая модель достоверной. Наиболее полной математической моделью является трехмерная модель, в которой учитываются нелинейные эфффекты.

Именно такая модель рассматривается в большинстве работ, посвященных исследованию напряженно-деформированного состояния безвоздушного колеса.

Так, работа [6] направлена на исследование сопротивлению качения безвоздушной шины с гибкими радиальными спицами и характера распределения давления в пятне ее контакта с опорной поверхностью. По результатам теоретических исследований с помощью разработанной вязкоупругой конечно-элементной математической модели установлено, что для уменьшения сопротивления качению и давления в контакте безвоздушной шины с опорной поверхностью необходимо увеличивать толщину опорного кольца и модуль сдвига эластичного полиуретана, из которого это кольцо изготовлено.

Исследование силовой неоднородности безвоздушной шины, а также вибраций и шума при ее качении выполнено в работе [7].

В работе [8] моделируется безвоздушная шина с гибкими спицами, опорное кольцо которой имеет работающий на сдвиг эластичный слой из пористого материала, армированного углеродистым волокном, и приводятся результаты теоретических исследований ее упругих свойств и сопротивления качению.

Предложена модель безвоздушной шины с гибкими спицами [9], опорное кольцо которой с осевыми полостями работает на сдвиг и изготовлено из алюминиевого сплава AL 7075-N6.

Метод конечных элементов был применен также и для численного моделирования безвоздушных шин с осевыми полостями гексагональной формы [10], а также безвоздушных колес с осевыми полостями трапецеидальной формы [11].

Однако следует отметить, что все эти безвоздушные колеса были изготовлены из армированной резины, что делает обоснованным применение метода конечных элементов с учетом нелинейных эффектов. При этом изготовление безвоздушных колес с помощью аддитивных технологий открывает возможности для построения упругих конечно-элементных моделей [12-13].

Метод конечных элементов был применен для расчета безвоздушного колеса в соответствии со структурной схемой, приведенной на рисунке 9 .

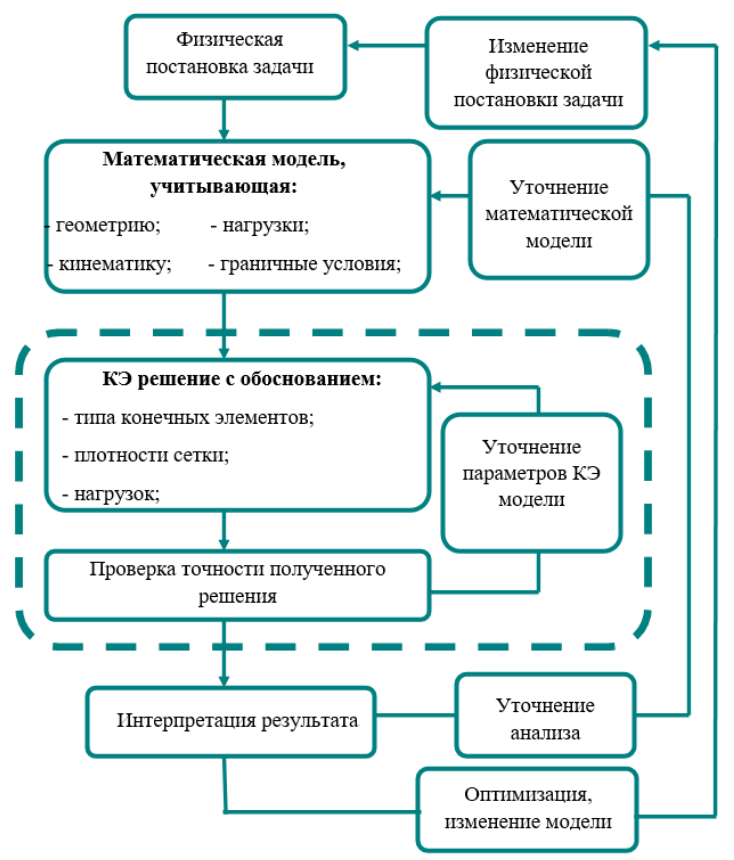

Рисунок 9 - Применение метода конечных элементов к расчету безвоздушного колеса 
Вестник Брестского государственного технического университета. 2021

В качестве среды разработки был выбран программный комплекс Solidworks.

Разработана твердотельная модель безвоздушного колеса, которая соответствует посадочным размерам электросамоката Xiaomi Mijia M365. При этом в качестве формы спиц была выбрана форма двойной спиральной пружины кручения. Данное решение обосновано необходимостью обеспечения боковой жесткости колеса при минимальном количестве деталей и узлов изделия. Применение витых пружин в подобных конструкциях ведет к необходимости внедрения направляющих в конечное изделие. Плоские витые пружины выполняют своей формой функцию бокового ребра жесткости [14].

Из выражений (6) и (7), полагая модули упругости спиц и обода равными, получаем:

$$
a^{2}=\frac{R^{2} F n}{2 \pi J}+1=\frac{\frac{\pi \cdot 0.2^{2}}{4} \cdot 36 \cdot 31^{2}}{2 \pi \cdot 0.3}+1=577.7 \rightarrow a=24.04 .
$$

Далее, согласно выражению (8) вычисляем:

$$
\alpha=\sqrt{\frac{24,04-1}{2}}=3,395, \quad \beta=\sqrt{\frac{24,04+1}{2}}=3,539 .
$$

Теперь находим

$$
\begin{gathered}
\operatorname{sh} \alpha \pi \approx \operatorname{ch} \alpha \pi \approx \frac{1}{2} e^{10,66}, \\
\sin \beta \pi=-0,992, \quad \cos \beta \pi=0,1223 .
\end{gathered}
$$

Согласно (10) имеем:

$$
A=-0,245 e^{-10,66}, \quad B=0,326 e^{-10,66} .
$$

Выражения (9) могут быть переписаны в виде:

$$
\begin{aligned}
& M=P\left(-0,00855+3,80 e^{-10,66} \operatorname{sh} \alpha \phi \cdot \sin \beta \phi-\right. \\
& \left.-5,05 e^{-10,66} \operatorname{ch} \alpha \phi \cdot \cos \beta \phi\right) \\
& P_{c}=P\left(0,0278+0,514 e^{-10,66} \operatorname{ch} \alpha \phi \cdot \cos \beta \varphi+\right. \\
& \left.+0,683 e^{-10,66} \operatorname{sh} \alpha \phi \cdot \sin \beta \phi\right) .
\end{aligned}
$$

Отсюда следует, что при небольших значениях $\varphi$ второе и третье слагаемое в скобках будут весьма малы и не будут существенно влиять на величину $M$ и $P_{c}$

Согласно (1) для водителя весом 77 кг и весе самоката 3 кг на заднее колесо приходится сила 40 кг. Тогда получаем, что $M_{\max }=88 \mathrm{kH}$, а наибольшее усилие на спицу $P_{c \max }=11,2$ кг.

Очевидно, что в полученном результате не учитывается предварительное натяжение спиц, которое задается при сборке, но оно не должно превышать по абсолютной величине $P_{c}$ max.

Из (11) следует, что

$$
P_{i_{\max }}=\frac{E F}{R} \omega_{\max },
$$

т. е. с учетом вышеприведенных вычислений

$$
\omega_{\max }=11,2 \cdot \frac{R}{E F} .
$$

Самокат Хiaomi Mijia M 365 не имеет амортизаторов, поэтому из (1) следует, что если мы рассмотрим радиус колеса $R$ как функцию двух переменных $(R(E, F))$, где $E$ - модуль упругости, учитывающий не только свойства материала, но и применяемую технологию печати и $F$ - площадь поперечного сечения спиц, то она не будет иметь экстремумов (частные производные первого порядка будут отличны от 0). Вместе с тем, можно рассмотреть задачу нахождения локальных экстремумов, существующих в допустимой области изменения $E$ и $F$.

Из (15) и вышеприведенных вычислений коэфффициентов следует, что в качестве целевой функции может быть выбрана функция

$$
68.2 \cdot H \rightarrow C_{\text {колеса }}
$$

где $C_{\text {колеса }}=150 \mathrm{H} / \mathrm{Mm}$.

Выполнен численный расчет, при этом установлено, что при $E=2140$ МПа и $H=2.2$ мм достигается решение поставленной задачи оптимизации.

С целью проверки результатов расчета модели был использован модуль исследования проектирования программного комплекса Solidworks. В результате проведенных исследований была создана расчетная модель с фиксированными посадочными размерами и свободными размерами геометрии спиц-демпфреров (рисунок 10-11). Были заданы статические и рабочие нагрузки, а также выполнен анализ деформированного состояния спиц обода в среде Simulation.

В качестве переменных параметров была использована толщина спицы. Радиусы $R_{1}$ и $R_{2}$ являются зависимыми от толщины спицы $H$ и перестраиваются автоматически. Всего проанализировано 43 возможных сценария с параметрами оптимизации - напряжения во всех точках детали меньше предела текучести при минимальной массе детали. Количество сценариев и время анализа напрямую связано с вычислительными возможностями ПЭВМ. Максимальная деформация при заложенных нагрузках составила 4 мм без разрушения.

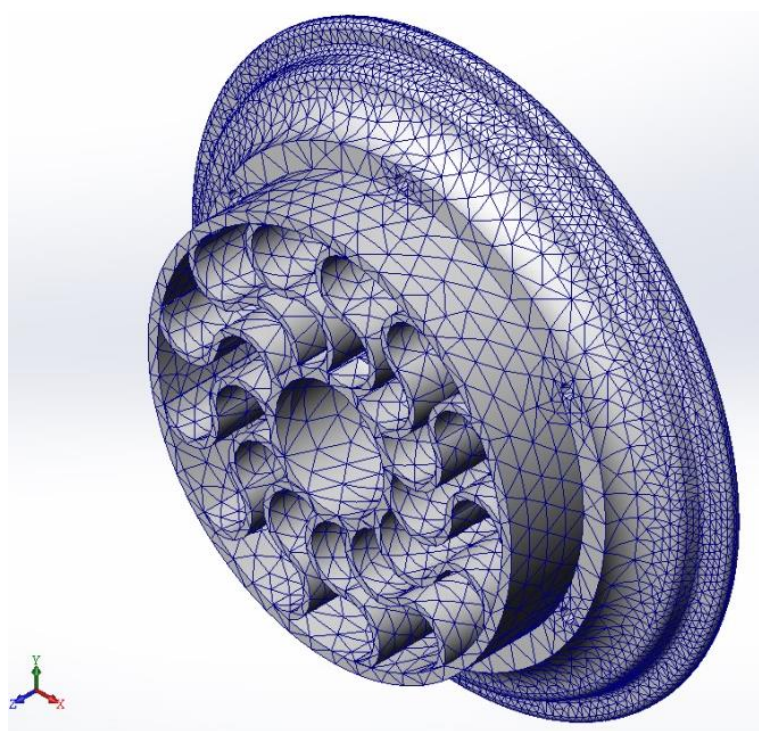

Рисунок 10 - Конечно-элементная модель безвоздушного колеса

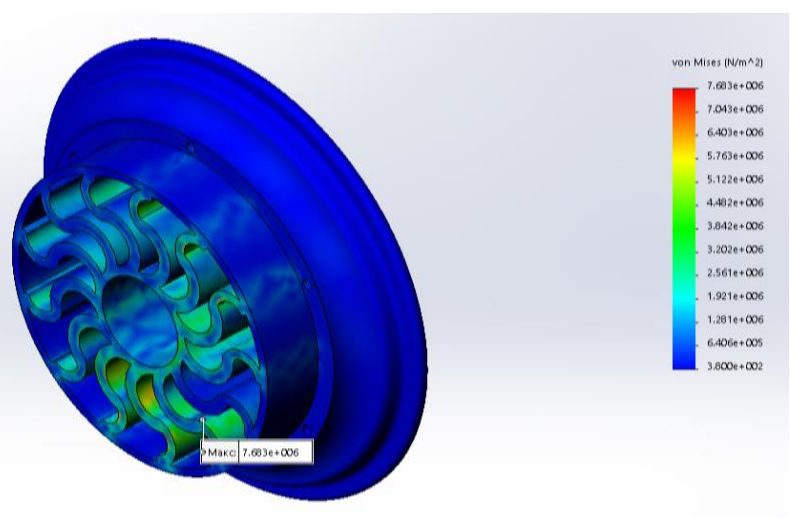

Рисунок 11 - Результат расчета спиц обода методом конечных элементов 


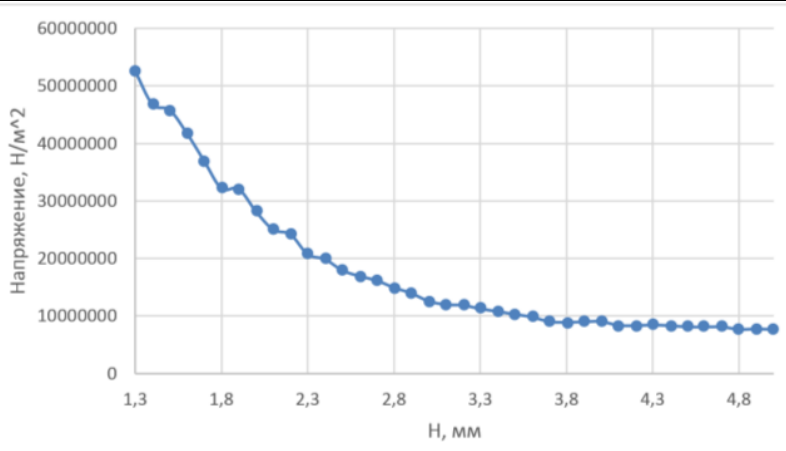

Рисунок 12 - Зависимости изменения максимальных напряжений от толщины спицы $H$

Разработанная трехмерная твердотельная модель безвоздушного колеса с использованием дискретно-континуального способа в методе конечных элементов с ориентацией на тетраэдрическую форму элементов сетки и фрагментацией исходной области позволяет организовать вычисления, характеризующиеся высокой точностью и достоверностью.

Таким образом, предложена методика расчета и конструирования безвоздушного колеса электрического самоката, основанная на применении системного подхода и включающая следующие этапы:

- анализ безвоздушного колеса как механической системы с учетом внешнего нагружения;

- расчет внешних нагрузок;

- расчет жесткости безвоздушного колеса и обоснование его конструктивного исполнения с помощью аналитических методов;

- создание модели безвоздушного колеса с помощью метода конечных элементов, ее оптимизация.

Разработанная методика позволит задать оптимальные конструкторско-технологические параметры для конструкции спиц безвоздушного колеса с внутренним демпфированием при переходе к синтезу конструкции, который целесообразно выполнять с помощью метода конечных элементов.

\section{Список цитированных источников:}

1. В России возник ажиотажный спрос на электросамокаты [Электронный ресурс] // Режим доступа: https://www.vedomosti.ru/newspaper/articles/2020/07/12/834402-azhiotazhnii-spros-na-elektrosamokati. - Дата доступа: 28.03.2020.

2. «Лишили прав - купил самокат». Истории минчан, для которых электросамокат стал транспортом [Электронный ресурс] // Режим доступа: https://news.tut.by/society/641244.html. - Дата доступа: 28.03 .2020 .

3. Феодосьев, В. И. Сопротивление материалов / В. И. Феодосьев. М. : Изд-во МГТУ им. Н. Э. Баумана, 1999. - 592 с.

4. Феодосьев, В. И. Избранные задачи и вопросы по сопротивлению материалов / В. И. Феодосьев. - М. : Наука, 1973, 400 с.

5. Методы анализа данных, оценивания и выбора в системных исследованиях / Сборник трудов. - М. : ВНИИСИ, 2006. - Вып.14. - 124 с.

6. Панкова, Л. А. Организация экспертиз и анализ экспертной информации / Л. А. Панкова, А. М. Петровский, М.В.Шнейдерман. М.:Наука, 2004. - 120 c.

7. Veeramurthy M., Ju J., Thompson L. L., Summers J. D. Optimization of geometry and material properties of a non-pneumatic tire for reducing rolling distance// International Journal of Vehicle Design. 2014. Vol. 66. No. 2. P 193-216. DOI: 10.1504/IJVD.2014.064567

8. Narasimhan A., Ziegert J.C., Thompson L.L. Effects of Material Properties on Static Load-Deflection and Vibration of a Non-Pneumatic Tire During High-Speed Rolling// SAE International Journal of Passenger Cars - Mechanical Systems. 2011. Vol. 4. No. 1. P. 59-72. DOI: 10. 4271/2011-01-0101

9. Ju J., Veeramurthy M., Summers J.D., Thompson L. Resistance of a Nonpneumatic Tire having a Porous Elastomer Composite Shear Band// Tire Science and Technology. 2013. Vol. 41. No. 3. P. 154-173.

10. Ma J., Summers J.D. Numerical Simulation of Tread Effects on the Interaction Between Cellular Shear Band Based Non-Pneumatic Tire and Sand// Proceedings of the ASME 2011 International Design Engineering Technical Conferences \& Computers and Information in Engineering Conference IDETC/CIE 2011. Washington, DC, USA. DOI: 10.1115/DETC2011-47044
11. Lee C., Ju J., Kim D.-M. Vibration Analysis of Non-Pneumatic Tires With Hexagonal Lattice Spokes// Proceedings of the ASME 2012 International Engineering Technical Conference \& Computers and Information in Engineering Conference IDETC/CIE 2012. Chicago, II., USA. DOI: 10.1115/DETC2012-70538

12. Kim K., Heo H., Uddin M.C., Ju J., Kim D.-M. Optimization of nonpneumatic Tire with Hexagonal Lattice Spokes for Reducing Rolling Resistance // SAE Technical Paper. 2015. DOI: 10.4271/2015-01-1515

13. Нигин, А. А. К расчету дисков сложной конфигурации методом конечных элементов / А. А. Нигин, В. К. Пушкарев // Расчеты на прочность. - М., 1980. - Вып. 21. - С. 15-19.

14. Автоматическое формирование сетки треугольных элементов для произвольных плоских областей / А. С. Цыбенко [и др.] // Проблемы прочности. - 1980. - № 12. - С. 84-89.

15. Бюл, В. Обзор методов формиролвания сетки конечных элементов / В. Бюл, Б. Буш // Тр. Амер. О-ва инж.-механиков. Сер. В. 1973. - T. 95, № 1. - C. 254-261.

16. Раздел 16. Упругие элементы в машинах [Электронный ресурс]. Режим доступа: http://www.detalmach.ru/lect13.htm/. - Дата доступа: 28.03.2020.

\section{References}

1. V Rossii voznik azhiotazhnyj spros na elektrosamokaty [Elektronnyj resurs] // Rezhim dostupa: https://www.vedomosti.ru/newspaper/articles/2020/07/12/834402-azhiotazhnii-spros-na-elektrosamokati. Data dostupa: 28.03.2020.

2. «Lishili prav - kupil samokat». Istorii minchan, dlya kotoryh elektrosamokat stal transportom [Elektronnyj resurs] // Rezhim dostupa: https://news.tut.by/society/641244.html. - Data dostupa: 28.03.2020.

3. Feodos'ev, V. I. Soprotivlenie materialov / V. I. Feodos'ev. - M. : Izdvo MGTU im. N. E. Baumana, 1999. - $592 \mathrm{~s}$.

4. Feodos'ev, V. I. Izbrannye zadachi i voprosy po soprotivleniyu materialov / V. I. Feodos'ev. - M. : Nauka, 1973, 400 s.

5. Metody analiza dannyh, ocenivaniya i vybora $v$ sistemnyh issledovaniyah / Sbornik trudov. - M. : VNIISI, 2006. - Vyp.14. - 124 s.;

6. Pankova, L. A. Organizaciya ekspertiz i analiz ekspertnoj informacii / L. A. Pankova, A. M. Petrovskij, M. V. SHnejderman. - M.:Nauka, 2004. $-120 \mathrm{~s}$.

7. Veeramurthy M., Ju J., Thompson L. L., Summers J. D. Optimization of geometry and material properties of a non-pneumatic tire for reducing rolling distance// International Journal of Vehicle Design. 2014. Vol. 66. No. 2. P 193-216. DOI: 10.1504/IJVD.2014.064567

8. Narasimhan A., Ziegert J.C., Thompson L.L. Effects of Material Properties on Static Load-Deflection and Vibration of a Non-Pneumatic Tire During High-Speed Rolling// SAE International Journal of Passenger Cars - Mechanical Systems. 2011. Vol. 4. No. 1. R. 59-72. DOI: 10. 4271/2011-01-0101

9. Ju J., Veeramurthy M., Summers J.D., Thompson L. Resistance of a Nonpneumatic Tire having a Porous Elastomer Composite Shear Band// Tire Science and Technology. 2013. Vol. 41. No. 3. P. 154-173.

10. Ma J., Summers J.D. Numerical Simulation of Tread Effects on the Interaction Between Cellular Shear Band Based Non-Pneumatic Tire and Sand// Proceedings of the ASME 2011 International Design Engineering Technical Conferences \& Computers and Information in Engineering Conference IDETC/CIE 2011. Washington, DC, USA. DOI: 10.1115/DETC2011-47044

11. Lee C., Ju J., Kim D.-M. Vibration Analysis of Non-Pneumatic Tires With Hexagonal Lattice Spokes// Proceedings of the ASME 2012 International Engineering Technical Conference \& Computers and Information in Engineering Conference IDETC/CIE 2012. Chicago, II., USA. DOI: 10.1115/DETC2012-70538

12. Kim K., Heo H., Uddin M.C., Ju J., Kim D.-M. Optimization of nonpneumatic Tire with Hexagonal Lattice Spokes for Reducing Rolling Resistance // SAE Technical Paper. 2015. DOI: 10.4271/2015-01-1515

13. Nigin, A. A. K raschetu diskov slozhnoj konfiguracii metodom konechnyh elementov / A. A. Nigin, V. K. Pushkarev // Raschety na prochnost'. - M., 1980. - Vyp. 21. - S. 15-19.

14. Avtomaticheskoe formirovanie setki treugol'nyh elementov dlya proizvol'nyh ploskih oblastej / A. S. Cybenko [i dr.] // Problemy prochnosti. -1980 . - № 12. - S. 84-89.

15. Byul, V. Obzor metodov formirolvaniya setki konechnyh elementov / V. Byul, B. Bush // Tr. Amer. O-va inzh.-mekhanikov. Ser. V. - 1973. T. 95, № 1. - S. 254-261.

16. Razdel 16. Uprugie elementy v mashinah [Elektronnyj resurs]. - Rezhim dostupa: http://www.detalmach.ru/lect13.htm/. - Data dostupa: 28.03.2020.

Материал поступил в редакцию 04.05.2021 